\title{
Large enhancement of the outer belt electrons during magnetic storms
}

\author{
Takahiro Obara $^{1}$, Yoshizumi Miyoshi ${ }^{2}$, and Akira Morioka ${ }^{2}$ \\ ${ }^{1}$ Communications Research Laboratory, Nukuikita, Koganei, Tokyo 184-8795, Japan \\ ${ }^{2}$ Planetary Plasma and Atmospheric Research Center, Tohoku University, Aoba, Aramaki, Aoba-ku, Sendai 980-8578, Japan
}

(Received January 11, 2001; Revised September 5, 2001; Accepted September 14, 2001)

\begin{abstract}
Major magnetic storm produces a big change in relativistic ( $>$ a few hundred keV) electron flux in the outer radiation belt. Although this phenomenon is well known, the characteristics of the storm that lead to the increase and the role of substorms in producing such energetic electrons are not well understood. In this paper, we examined the transport and energization of outer belt electrons on the basis of NOAA and Akebono measurements for early November, 1993 magnetic storm. The result demonstrated that the intermediate-energy (30-100 keV) electrons in the plasma sheet were injected to the near-Earth $(L=3-4)$ region during the storm main phase, and an increase of $\mathrm{MeV}$ electrons took place due to the internal acceleration of the source electrons. We have, then, checked the counter example, the January 1997 magnetic storm, in which we saw a strong injection of 30-100 $\mathrm{keV}$ electrons but no intensification of relativistic energies. Following processes were requested in intensifying relativistic electrons in the outer radiation belt; i.e. the supply of source electrons with intermediate-energy (30$100 \mathrm{keV}$ ) and a possible acceleration of them. Hint of the acceleration would be the strong magnetic activities during the storm recovery phase. We are speculating that substorm-related electric and magnetic field fluctuations and/or waves would accelerate electrons efficiently. Even though the present work is a case study, it demonstrates that the internal acceleration is actually taking place and it leads a large enhancement of the relativistic electrons in the outer radiation belt.
\end{abstract}

\section{Introduction}

Dramatic changes in the outer radiation belt electrons during magnetic storms were first observed in the early 1960's: the electron flux at the geostationary orbit altitude decreases rapidly at the onset of the main phase of a magnetic storm. Follow-up analysis using SAMPEX and GPS observations was made by Li et al. (1997), and they showed that the decrease occurs not only at geostationary orbit but also near the peak position of the outer radiation belt $(L \sim 4)$. It has been reported that an increase in the number of relativistic electrons to above the pre-storm level occurs in the outer radiation belt during the magnetic storm (Li et al., 1997; Obara et al., 2000a and references therein). They found that the flux of electrons appeared first in low $L$-shell regions and at lower energies and the flux in higher $L$ shells increased with a significant time delay. Nakamura et al. (1998) used SAMPEX observations to examine an adiabatic deceleration/acceleration process and concluded that additional supply and/or acceleration process must, in fact, be responsible for the increase of relativistic electrons in the outer radiation belt.

It has been an important outstanding problem over 40 years how the source populations are being supplied in the outer radiation zone during a magnetic storm and how they are energized. It is a tough problem but we like to try to understand them by referring the recent Akebono and NOAA electron observations. The purposes of the present paper are

Copy right(c) The Society of Geomagnetism and Earth, Planetary and Space Sciences (SGEPSS); The Seismological Society of Japan; The Volcanological Society of Japan; The Geodetic Society of Japan; The Japanese Society for Planetary Sciences.
(1) to identify the evidence of the internal acceleration, and (2) to specify the deep injection of source electrons by the substorm. By paying a particular attention to substorm activities, we want to consider a possible explanation for the outer belt re-building during the storm recovery phase.

\section{NOAA Energetic Electron Observations}

On the NOAA satellite a particle detector has been installed, observing local trapped electrons in three energy channels $E_{1}(>30 \mathrm{keV}), E_{2}(>100 \mathrm{keV})$, and $E_{3}(>300$ $\mathrm{keV})$. In early November 1993, the NOAA 12 satellite carried out observations near the morning-evening meridian sector. An averaged count rate in both morning and evening local times is used to demonstrate the intensity of electrons every pass, as shown in Figs. 1(a) and 1(b). When the satellite traverses in the southern hemisphere, it observes a large variation of electron fluxes, which shows a strong regional dependence. To see the temporal variation more clearly we have only used northern hemisphere data. The top panel in each figure shows the $L-t$ diagram for number of electrons, and the variations in $\mathrm{Kp}$ and Dst are given in the middle and bottom panels, respectively. The count rate as measured by the $E_{3}$ detector ( $>300 \mathrm{keV}$ ) is given in Fig. 1(a) and the data from the $E_{1}$ detector ( $>30 \mathrm{keV}$ ) is shown in Fig. 1(b), where the vertical axes demonstrate the McIlwain- $L$ value and the horizontal axes show universal time (UT), covering the period from November 2 (day 306) to November 9 (day 313) in 1993 .

In Fig. 1(a) we can see that a large increase in the number of $>300 \mathrm{keV}$ electrons occurred around noon of Novem- 

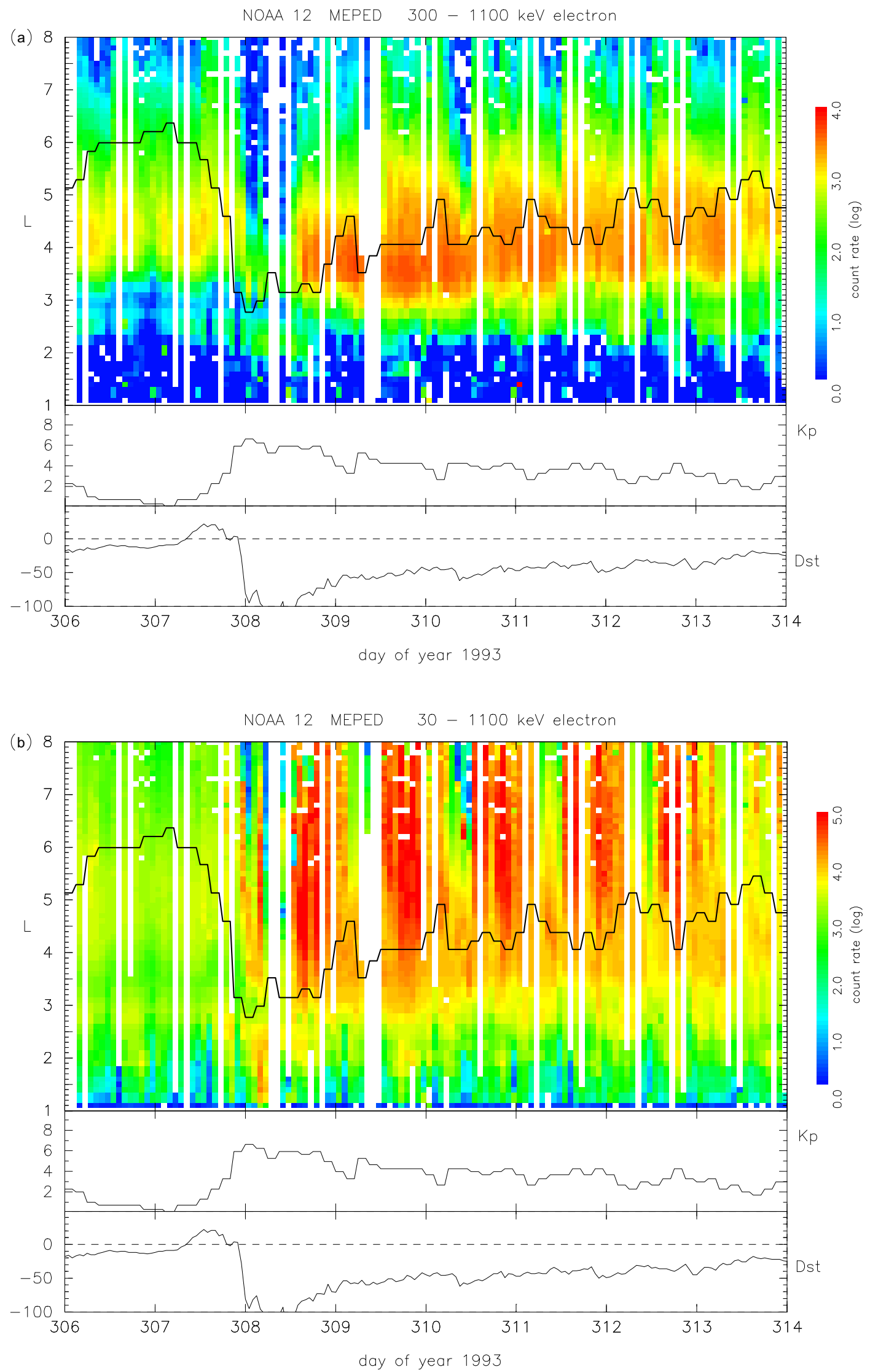

Fig. 1. NOAA energetic electron observations; (a) $L-t$ diagram for electrons with energies of $>300 \mathrm{keV}$ (top), Kp index variation (middle) and Dst index variation (bottom), for the interval from November 2 (day 306 of the year) to November 9 (day 313 ) in 1993 . (b) $L-t$ diagram for electrons with energies of $>30 \mathrm{keV}$ (top), Kp index variation (middle) and Dst index variation (bottom), for the interval from November 2 (day 306 ) to November 9 (day 313 ) in 1993 . 

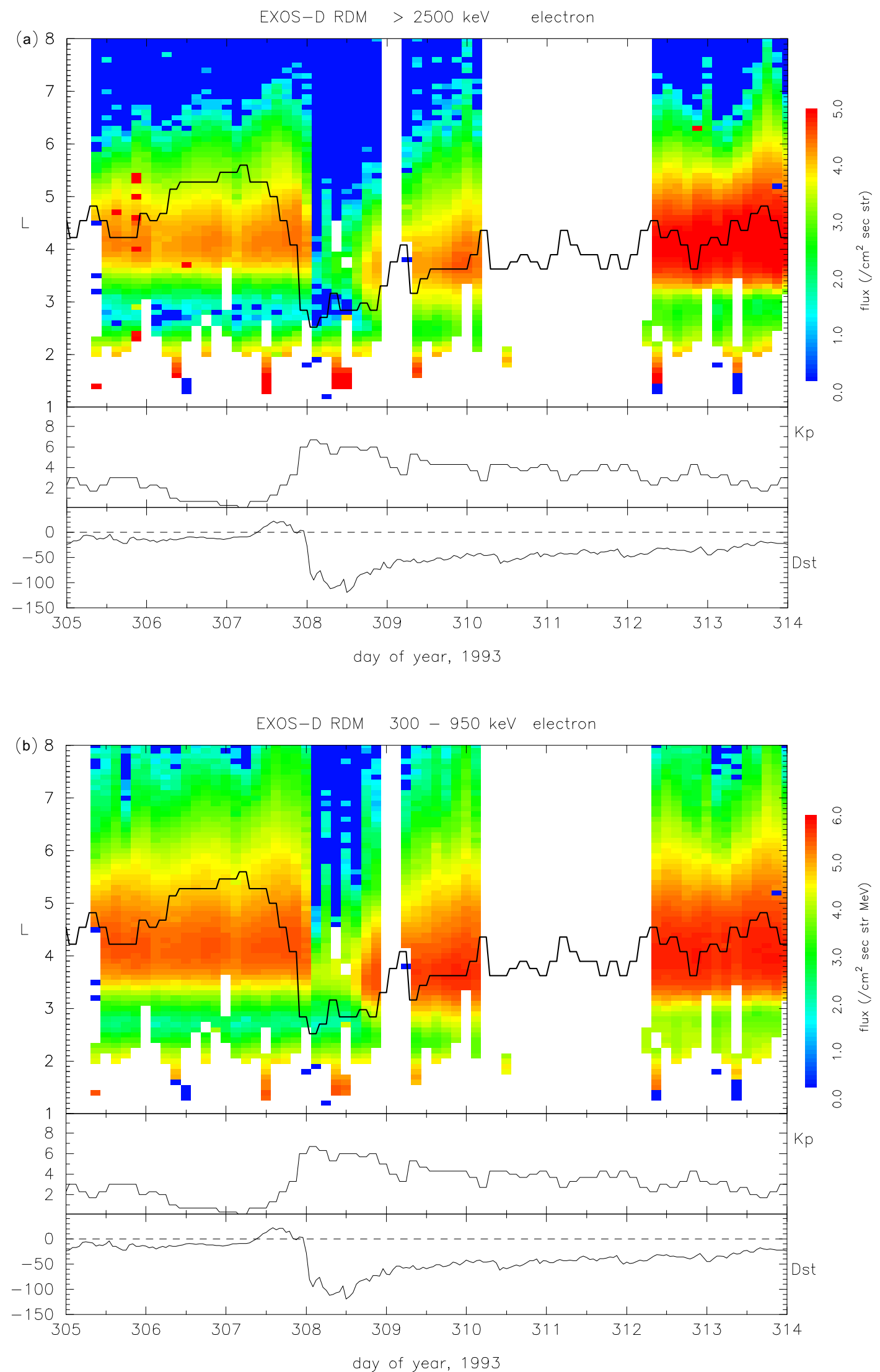

Fig. 2. Akebono highly energetic electron observations; (a) $L$ - $t$ diagram for electrons with energies of $>2.5 \mathrm{MeV}$ (top), Kp index variation (middle) and Dst index variation (bottom), for the interval from November 1 (day 305) to November 9 (day 313) in 1993. (b) $L-t$ diagram for electrons with energies from $300 \mathrm{keV}$ to $900 \mathrm{keV}$ (top), Kp index variation (middle) and Dst index variation (bottom), for the interval from November 1 (day 305) to November 9 (day 313) in 1993. 

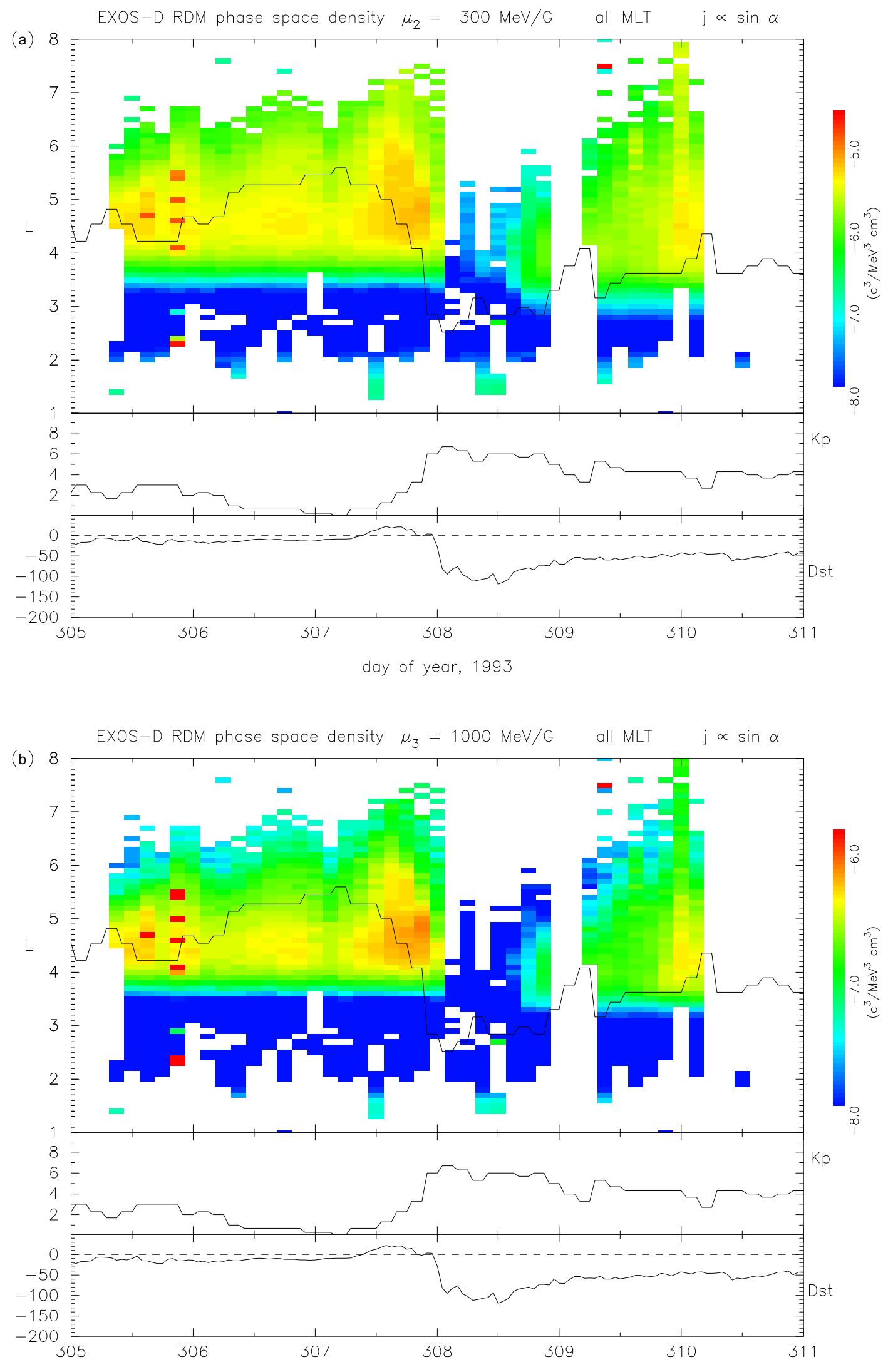

day of year, 1993

Fig. 3. Phase space density at $\mu=300 \mathrm{MeV} / \mathrm{G}$ (a) and $\mu=1000 \mathrm{MeV} / \mathrm{G}$ (b), deduced from Akebono observations. Middle and bottom panels in both figures represent $\mathrm{Kp}$ and Dst indexes. 
ber 4 (day 308), and persisted for a long time, showing a gradual expansion with regard to $L$-value and the peak location also shifted outward smoothly. On closer inspection of Fig. 1(b) we can identify several particle injections. The first injection took place at the onset of the main phase (around 23 UT on November 3 (day 307)). This injection stopped around $L \sim 3.5$, or in that range. The second injection occurred around the noon of November 4 (day 308), and it was strongest. It actually transported $>30 \mathrm{keV}$ electrons as low as $L \sim 3.0$ or so. Injection continued to take place quasi-periodically throughout the recovery phase. By closer inspection of injection events, electrons were transported as low as $L \sim 4$ or so. The lifetime of such energy electrons could be a few hours and time intervals of injections were more than ten hours. We, therefore, regard these injections are real. To see whether they were actually real injections, we further refereed independent observations of energetic electrons at geostationary orbit. According to LANL observations in the nightside sector, there were injections around 13 UT (day 308), 10 UT (day 309), 10 UT and 19 UT (day 310), 13 UT and 19 UT (day 311), and 11 UT (day 312). There was no injection on day 313 . Hence, we like to conclude that NOAA 12 satellite actually observed the injections. As time progressed, the injection was weakened and the injection boundary gradually moved outward. The thick lines in Figs. 1(a) and 1(b) represent the plasmapause location determined by the model based on Kp. The movement of the plasmapause seems somewhat consistent with the injection boundary during the storm recovery phase.

\section{Akebono Relativistic Electron Observations}

The Akebono satellite was launched on February 22 in 1989 into an initial orbit with an inclination of 75 deg., an apogee of $10500 \mathrm{~km}$ and a perigee of $275 \mathrm{~km}$. A radiation monitor (RDM) was installed to monitor the radiationparticle environment. The RDM consists of four solid-state detectors that cover electrons in three energy ranges; $0.3-$ 0.95 MeV, 0.95-2.5 MeV, and more than 2.5 MeV. Akebono satellite traversed the evening sector (MLT $\sim 17 \mathrm{~h}$ ) in early November 1993 and measured the outer belt electrons at altitudes of $5000-6000 \mathrm{~km}$. The top panel in Fig. 2(a) shows the distribution of $>2.5 \mathrm{MeV}$ electrons as a function of McIlwain- $L$ parameter, and the variations in Kp and Dst are given in the middle and bottom panels, respectively. The figure covers 9 days (day 305 to day 313), but we have a data gap on days 310 and 311 , which is due to a satellite anomaly.

On day 307 (November 3) we can clearly identify the outer belt and a slot. The peak portion of the outer belt was located at $L=4$ and the slot was around $L=2.7$. At the commencement of the magnetic storm at 23 UT on day 307 (Knipp et al., 1998), the flux in the outer belt dropped dramatically. A large increase in the number of energetic electrons in the outer radiation belt is evident in the afternoon of day 308, where the increase took place closer to the Earth $(L=3.5)$. A detailed description has already been given by Obara et al. (2000a), and the essential point of Akebono observations is a large evolution of the outer radiation belt during the recovery phase of the magnetic storm. The peak intensity of relativistic electrons increased with time and the outer belt expanded outward quite smoothly. By day 313 (November 9) the number of $>2.5 \mathrm{MeV}$ electrons surpassed its pre-storm level at geostationary $L$ shells.

In Fig. 2(b) we have plotted Akebono observations of electrons with intermediate-energy (300-900 keV). Format is same as Fig. 2(a). Compared with higher energy electrons shown in Fig. 2(a), the distribution of 300-900 keV electrons seem wider, but the dynamic variation looks similar to $>2.5 \mathrm{MeV}$ electrons. At the commencement of the magnetic storm, the intensity of 300-900 keV electrons decreased rapidly. It is noticed that an increase of 300-900 $\mathrm{keV}$ electrons in the heart of the outer belt started a little bit faster than that of $>2.5 \mathrm{MeV}$ electrons (Obara et al., 2000a).

In the previous paper (Obara et al., 2000a) we have made speculation that "seed" electrons with intermediate-energy should be transported during the storm main phase and they should be accelerated internally, causing outer belt rebuilding. This scenario (Obara et al., 2000a) is preliminary and it actually needs follow-up study. We, therefore, calculated phase space density at constant $\mu$ by using Akebono observations for three different energies with pitch angle information. Results are given in Figs. 3(a) and 3(b). In the top of Fig. 3(a) distribution of phase space density at $\mu=300$ $\mathrm{MeV} / \mathrm{G}$ is shown. At the commencement of the main phase (23 UT on day 307), we can see a sharp decrease of the phase space density in the outer radiation belt. This disappearance lasted for 10 hour or so, and an increase was found in the heart of the outer belt around 12 UT on day 308. Over two days the intensity of the phase space density increased with time. By the end of day 309 the intensity reached its pre-storm level. When we look more energetic population ( $\mu=1000 \mathrm{MeV} / \mathrm{G}$ ) as shown in Fig. 3(b), it took more time for the phase space density to recover, but the characteristics in the increase was in the same manner.

In order to see it more clearly, we plotted the time evolution of the phase space density at $\mu=300 \mathrm{MeV} / \mathrm{G}$ and $\mu=1000 \mathrm{MeV} / \mathrm{G}$. Results were given in Fig. 4, where the PSD at $L=4$ is show in panel (a) and the PSD at $L=5$ is given in panel (b). It is worth mentioning that the increase is rapid in lower $L$ region $(L=4)$ rather than far region ( $L=5)$; actually the rate of the increase is twice at $L=4$ than that at $L=5$. At $L=4$, the intensity of the PSD at $\mu=300 \mathrm{MeV} / \mathrm{G}$ reached $10^{-6}\left(\mathrm{c}^{3} / \mathrm{MeV}^{3} \mathrm{~cm}^{3}\right)$ within one day (day of 308). Suppose the increase is nearly constant, a rate of increase is about $10^{-11} \mathrm{c}^{3} / \mathrm{MeV}^{3} \mathrm{~cm}^{3} \mathrm{sec}$, which would account for the internal acceleration. From both Figs. 3 and 4 it seems valid that the internal acceleration took place in the outer radiation belt during the recovery phase of this magnetic storm.

\section{Discussions}

We have examined early November 1993 magnetic storm to identify the source population and to investigate the acceleration process within the outer belt. The main points of this paper are to show the injection of intermediate-energy (30-100 keV) electrons based on the NOAA observations and to show the evidence of internal acceleration by using Akebono energetic electron observations. In the November 1993 magnetic storm, there was a prolonged magnetic activity during the recovery phase, in which some acceleration 
(a)

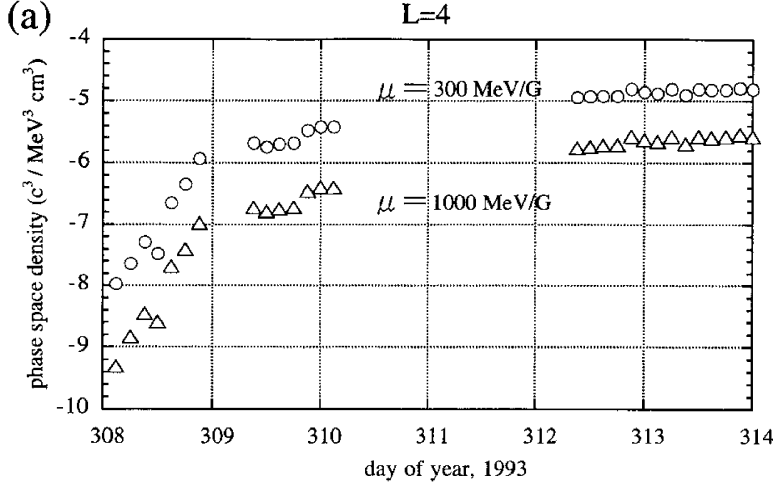

(b)

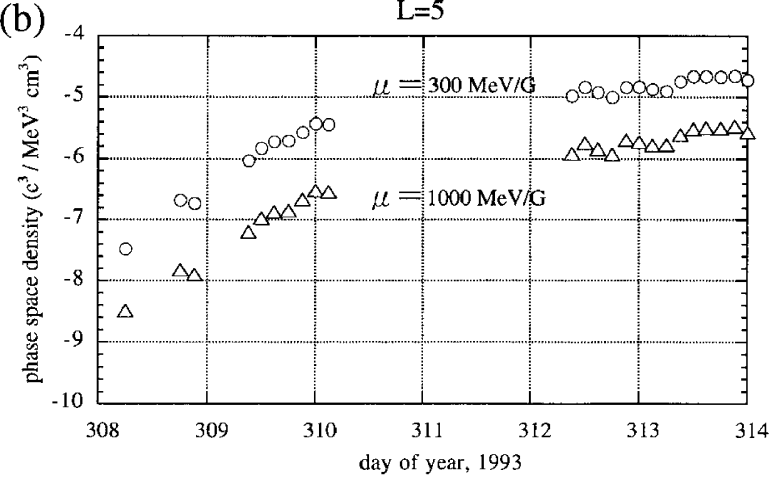

Fig. 4. Variation of phase space density at $L=4$ (a) and $L=5$ (b). Increase of the phase space density at $L=5$ took longer time than that at $L=4$.

took place in the heart of the outer belt. There have been works, which insist internal acceleration by means of ULF waves (Elkington et al., 1999, and references therein) and ELF/VLF waves (Horne and Thorne, 1998 and references therein). We leave the check of the individual process, but such wave activities should be intensified due to the magnetic activities during the storm recovery phase. Hint has been presented in Obara et al. (1998). They compared the relativistic electron flux at geostationary orbit with the interplanetary magnetic field (IMF) orientation, and found that a large flux enhancement occurs when the IMF is directing southward during the storm recovery phase. In the November 1993 magnetic storm, the IMF mostly directed southward during the storm recovery phase (Knipp et al., 1998), resulting in large magnetic activities during the storm recovery phase.

It should be needed to find out very counterpart example. January 1997 magnetic storm would be one example, we believe. On January 10, 1997 an interplanetary shock hit the Earth. After the shock, the IMF rotated southward ( -15 nT) at around $06 \mathrm{UT}$, and this caused the magnetic storm. IMF then rotated northward over a period of about one day, changing its sign around 18 UT on January 10 (Reeves et al., 1998). Dst reached its minimum level at around $10 \mathrm{UT}$ on January 10 and increased monotonically from there. The level of magnetic activity after that was quite low, due to the prolonged northward IMF condition. Figures 5(a) and 5(b) show NOAA observations of $>300 \mathrm{keV}$ and $>30 \mathrm{keV}$ electrons, respectively, covering the interval from January 7 to
15. Around 10 UT on January 10 we can see a remarkable increase in the number of $>300 \mathrm{keV}$ electrons. The increase in the number of the energetic electrons was well documented by Li et al. (1998). There was a great substorm activity around 11 UT. In Fig. 5(b) we can see a big injection of $>30 \mathrm{keV}$ electrons around that time. Actually the supply of seed electrons went as low as $L \sim 3.8$ or so. An increase of $>300 \mathrm{keV}$ electrons also occurred around 12 UT. It should be noted that there was little injection after the noon of January 10, which was due to the relative quiescence of the magnetic activity. It is important mentioning that the peak flux level took rather low constant value over the period from January 11 to January 15, showing no significant increase in the relativistic electrons in the outer belt.

Returning to the roles of the storm-time substorm, substorms in the main phase should provide a supply of source electrons. According to the NOAA observations, injections of electrons into the heart of the outer radiation belt took place during the main phase of the magnetic storms. The injection had stopped by around $L \sim 3.8$ in the case of the January 1997 magnetic storm, but reached much closer to the Earth for November 1993 magnetic storm. The location of the inner limit seems to be related to the magnitude of the storm (Obara et al., 2000b).

The acceleration process is important and essential for outer belt re-building. Baker et al. (1998a, b) demonstrated evidence for electron acceleration in the magnetosphere on the basis of SAMPEX and POLAR observations in combination with ground-based magnetometer measurements. For the November 1993 magnetic storm, supply of the seed electrons continued over the storm recovery period. The depth to which seed electrons were transported became less and less as time progressed. To increase the number of relativistic electrons in higher $L$ regions $(L=5 \sim 8)$ additional acceleration is needed. Based on Akebono observations we have estimated recovering rate of high energetic electrons in the outer radiation belt during the storm recovery phase. The rate is about $10^{-11} \mathrm{c}^{3} / \mathrm{MeV}^{3} \mathrm{~cm}^{3} \mathrm{sec}$ in the heart of the outer radiation belt. Even though this value depends on the condition of each storm, this value should be the good quantitative target for the theory to evaluate the acceleration processes not only in the heart of outer radiation belt but also higher $L$ regions.

Lastly, it should be noted that outward radial diffusion might contribute outward expansion of the outer belt. We can actually see large outward expansion of the outer radiation belt in November 1993 magnetic storm (see Fig. 2). Works with regards to the radial diffusion have been done by many authors (e.g. Selesnick and Blake, 2000 and references therein). The direction of the diffusion should be decided by the exact spatial distribution of the phase-space density as a function of the adiabatic invariants. In case of the internal acceleration, there will be a spatial peak of the phase space density in the outer radiation belt, which leads both inward and outward radial diffusion. Since this paper could point out some characteristics with regard to the internal acceleration, next step of the research works would be to investigate diffusion coefficient and estimate an acceleration efficiency etc. 


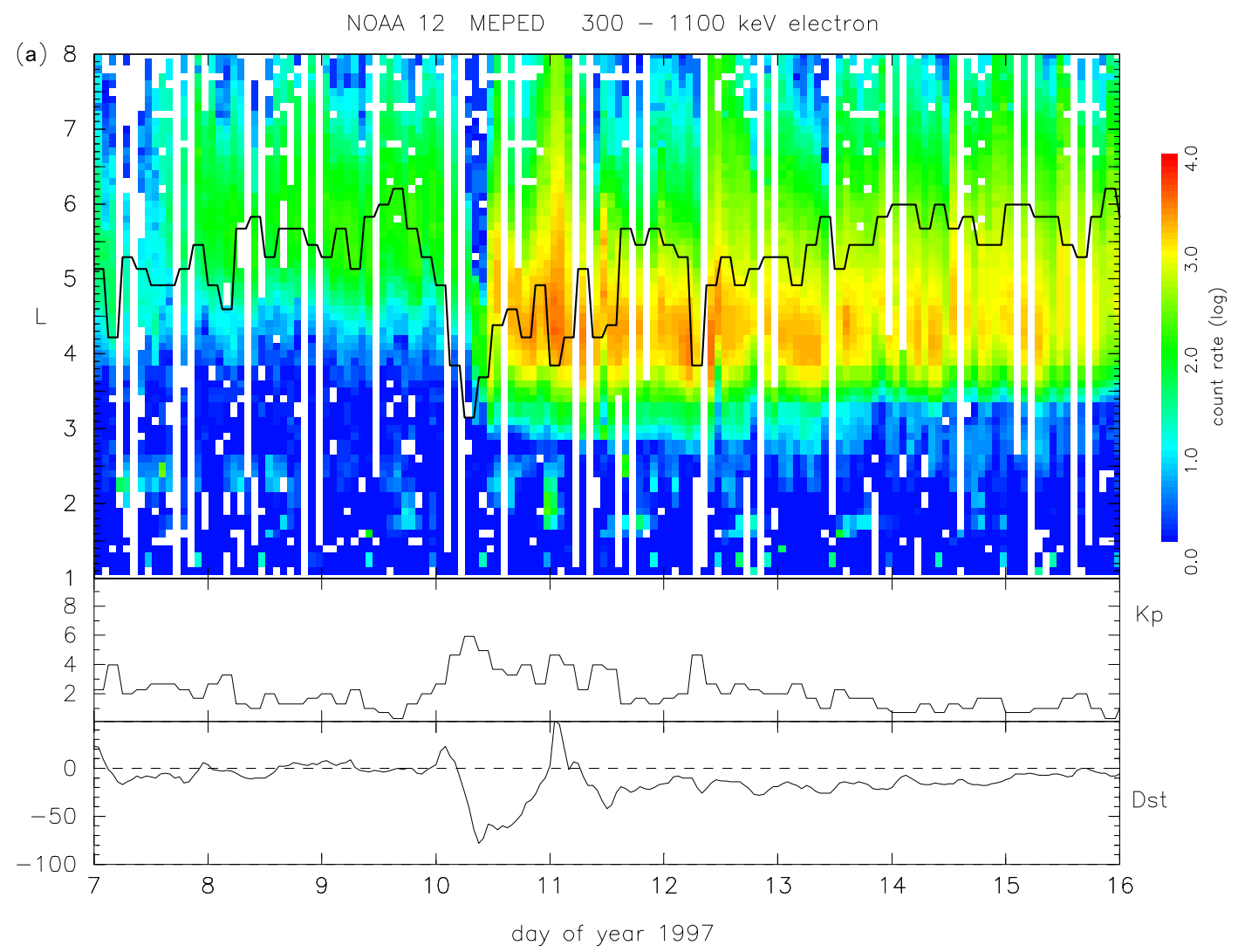

NOAA 12 MEPED $30-1100 \mathrm{keV}$ electron

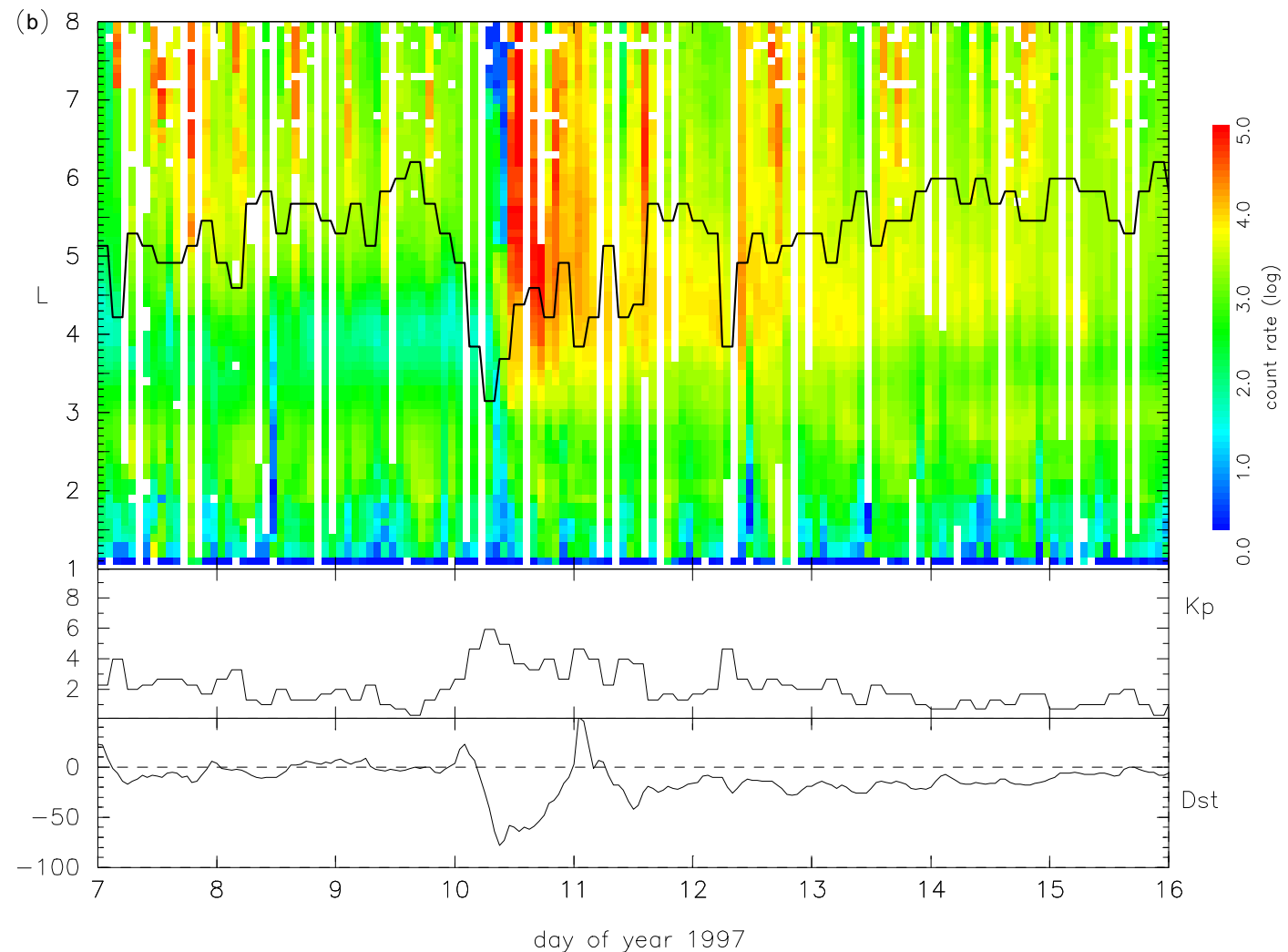

Fig. 5. NOAA energetic electron observations: (a) $L-t$ diagram for electrons with energies of $>300 \mathrm{keV}$ (top), Kp index variation (middle) and Dst index variation (bottom), for the interval from January 7 to January 15 in 1997 . (b) $L$ - $t$ diagram for the electrons with energies of $>30 \mathrm{keV}$ (top), Kp index variation (middle) and Dst index variation (bottom), for the interval from January 7 to January 15 in 1997. 
Acknowledgments. We are grateful to Dr. T. G. Onsager, and Dr. T. Nagatsuma for the valuable suggestions, and Dr. G. D. Reeves for allowing us to refer LANL observations. The research described in the present paper used the Akebono Science Data Base (SDB) of the Inst. of Space and Astronautical Sci. (ISAS) of Japan. NOAA particle data were supplied by the NOAA data center.

\section{References}

Baker, D. N., T. I. Pulkkinen, X. Li, S. G. Kenekal, J. B. Blake, R. S. Selesnick, M. D. Henderson, G. D. Reeves, H. E. Spence, and G. D. Rostoker, Coronal mass ejections, magnetic clouds, and relativistic magnetospheric electron events: ISTP, J. Geophys. Res., 103, 17279-17291, 1998a.

Baker, D. N., X. Li, J. B. Blake, and S. G. Kenekal, Strong electron acceleration in the Earth's magnetosphere, Adv. Space Res., 21, 609$613,1998 b$

Elkington, S. R., M. Hudson, and A. A. Chan, Acceleration of relativistic electrons via drift-resonant interaction with toroidal-mode Pc-5 ULF oscillations, Geophys. Res. Lett., 26, 3273-3276, 1999.

Horne, R. B. and R. M. Thorne, Potential waves for relativistic electron scattering and stochastic acceleration during magnetic storms, Geophys. Res. Lett., 25, 3001-3004, 1998.

Knipp, D. J. et al., An over view of the early November 1993 geomagnetic storm, J. Geophys. Res., 103, 26197-26220, 1998.

Li, X., D. N. Baker, M. Temerin, T. E. Cayton, G. D. Reeves, R. A. Christensen, J. B. Blake, M. D. Looper, R. Nakamura, and S. G. Kenekal, Observations of the outer zone electron variation during the November 3-4, 1993, magnetic storm, J. Geophys. Res., 102, 14123-14140, 1997.

Li, X., D. N. Baker, M. Temerin, T. Cayton, G. D. Reeves, T. Araki, H.
Singer, D. Larson, R. P. Lin, and S. G. Kanekal, Energetic electron injections into the inner magnetosphere during the Jan. 10-11, 1997 magnetic storm, Geophys. Res. Lett., 25, 2561-2564, 1998.

Nakamura, R., K. Kamei, and Y. Kamide, SAMPEX observation of stormassociated electron flux variation in the outer radiation belt, J. Geophys. Res., 103, 26261-26269, 1998.

Obara, T., T. Nagatsuma, and T. G. Onsager, Effects of the interplanetary magnetic field (IMF) on the rapid enhancement of relativistic electrons in the outer radiation belt during the storm recovery phase, in SUBSTORM4, edited by S. Kokubun and Y. Kamide, pp. 215-218, Terra Scientific Publishing Company, 1998.

Obara, T., T. Nagatsuma, M. Den, Y. Miyoshi, and A. Morioka, Main-phase creation of "seed" electrons in the outer radiation belt, Earth Planets Space, 52, 41-47, 2000a.

Obara, T., M. Den, Y. Miyoshi, and A. Morioka, Energetic electron variation in the outer radiation zone during early May 1998 magnetic storm, JASTP, 65, 1407-1412, 2000b.

Reeves, G. D., D. N. Baker, R. D. Belian, J. B. Blake, T. E. Cayton, J. F. Fennel, R. H. W. Fridel, M. M. Meier, R. S. Selesnick, and H. E. Spence, The global response of relativistic radiation belt electrons to January 1997 magnetic cloud, Geophys. Res. Lett., 25, 3265-3268, 1998.

Selesnick, R. S. and J. B. Blake, On the source location of radiation belt relativistic electrons, J. Geophys. Res., 105, 2607-2624, 2000.

T. Obara (e-mail: T.Obara@crl.go.jp), Y. Miyoshi (e-mail: miyoshi@ pparc.geophys.tohoku.ac.jp), and A. Morioka (e-mail: morioka@pparc. geophys.tohoku.ac.jp) 